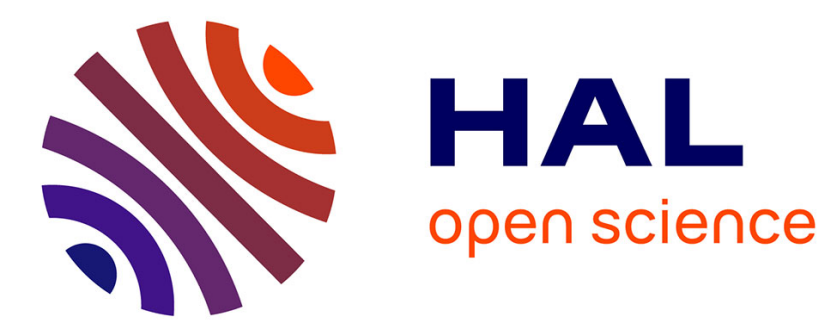

\title{
Remarques à propos des demandes d'analyses par le Carbone 14 et l'interprétation de leurs résultats
}

Georgette Delibrias, Jacques Évin

\section{To cite this version:}

Georgette Delibrias, Jacques Évin. Remarques à propos des demandes d'analyses par le Carbone 14 et l'interprétation de leurs résultats. Bulletin de la Société préhistorique française, 1972, 69 (6), pp.189

- 192. 10.3406/bspf.1972.4376 . hal-03403690

\author{
HAL Id: hal-03403690 \\ https://hal.science/hal-03403690
}

Submitted on 26 Oct 2021

HAL is a multi-disciplinary open access archive for the deposit and dissemination of scientific research documents, whether they are published or not. The documents may come from teaching and research institutions in France or abroad, or from public or private research centers.
L'archive ouverte pluridisciplinaire HAL, est destinée au dépôt et à la diffusion de documents scientifiques de niveau recherche, publiés ou non, émanant des établissements d'enseignement et de recherche français ou étrangers, des laboratoires publics ou privés. 


\section{Remarques à propos des demandes d'analyses par le Carbone 14} et l'interprétation de leurs résultats

Georgette Delibrias, Jacques Evin

\section{Citer ce document / Cite this document :}

Delibrias Georgette, Evin Jacques. Remarques à propos des demandes d'analyses par le Carbone 14 et l'interprétation de leurs résultats. In: Bulletin de la Société préhistorique française. Comptes rendus des séances mensuelles, tome 69 , n6, 1972. pp. 189-192;

doi : https://doi.org/10.3406/bspf.1972.4376

https://www.persee.fr/doc/bspf_0249-7638_1972_num_69_6_4376

Fichier pdf généré le 08/09/2020 


\title{
Remarques à propos des demandes d'analyses
}

\section{par le carbone 14 et l'interprétation de leurs résultats}

\author{
par G. Delibrias $\left({ }^{\star}\right)$ et J. Evin $\left(^{* *}\right)$
}

La méthode de datation par le carbone 14, mise au point dès 1952 , a été largement exploitée depuis cette époque. Le nombre et l'importance des laboratoires de Carbone 14 vont toujours croissant ; les techniques utilisées se diversifient ; mais quels que soient les progrès accomplis dans ce domaine, les mesures d'âge qui sont des mesures de radioactivité à très bas niveaux, sont longues, difficiles et par conséquent coûteuses. Chacune d'elles représente une quantité importante de travail et nécessite l'immobilisation d'appareils complexes. De ce fait, les possibilités de mesures restent et resteront probablement encore longtemps limitées. Il convient d'en tirer parti le plus judicieusement possible. Tout ne pourra donc pas être daté sans discernement, ce qui signifie que chercheurs et laboratoires de C-14 seront souvent appelés à faire un choix parmi les nombreux problèmes posés et les multiples prélèvements effectués. Ce choix sera fait avec le souci constant de traiter en priorité les problèmes les plus importants, de sélectionner les échantillons les plus représentatifs au point de vue archéologique et ceux dont la datation sera la plus significative.

\section{Choix des échantillons.}

Il est tout d'abord évident que la datation d'un échantillon doit correspondre à une question posée, doit résoudre un problème scientifique bien précis : la datation pour la datation d'un objet quelconque trouvé par hasard, en dehors

\footnotetext{
(*) Centre des Faibles Radioactivités, C.N.R.S. Gif-surYvette (91).

(**) Laboratoire de Radiocarbone, Département de Géologie, Cniversité Iyon I. Institut de Physique Nucléaire, 43, bd du 11-Novembre, 69 - Villeurbanne.
}

de tout contexte, est sans intérêt. Les mesures ne doivent intervenir en fait que lorsqu'une étude archéologique, palynologique ou géologique, conduite sur le site, est déjà parvenue à un stade tel qu'elle permet de circonscrire un événement caractéristique à dater. Citons, par exemple, le cas de datations prématurées d'une tourbière, effectuées de façon systématique en fonction de la profondeur, tous les $20 \mathrm{~cm}$, or l'étude palynologique peut faire apparaître entre les niveaux étudiés des accidents caractéristiques qu'il aurait fallu dater avec précision, ou une évolution rapide qu'il aurait été intéressant de suivre à l'aide de mesures plus rapprochées alors que le reste du profil ne justifiait pas une telle fréquence de mesures. Il peut en être de même en archéologie où certaines parties d'un gisement, fouillées plus tardivement, peuvent s'avérer plus intéressantes à dater que celles dégagées en début de campagne.

Toutefois la datation d'un échantillon judicieusement choisi au cours de fouilles importantes peut être exceptionnellement effectuée très rapidement dans le but d'orienter les recherches. Mais, toujours dans le souci de ne pas encombrer indûment les laboratoires, ces mesures urgentes ne doivent être demandées que si elles peuvent effectivement guider la recherche sur le terrain.

Enfin le problème se pose souvent de savoir si l'échantillon est «datable »; autrement dit, si la méthode de datation par le radiocarbone peut être utilisée dans ce cas. Le laboratoire de C-14 est alors seul juge en la matière. On peut cependant donner ici les critères principaux qui interviennent dans ce jugement. Rappelons que ne peuvent être datés que les matériaux carbonés dont le carbone provient en totalité, et récemment, de l'atmosphère, au moment de la forma- 
tion de ces matériaux (1) ; tout apport de carbone qui n'est pas dans ce cas est considéré comme une pollution qui peut rendre l'échan. tillon impropre à la datation. Les sources essenticlles de pollution sont parfois bien visibles : présence de radicelles qui rajeunissent l'échantillon, ou au contraire peu apparentes : apport de carbone minéral qui le vieillit. Le nettoyage à la pince et les traitements chimiques effectués au laboratoire tendent à éliminer ces pollutions ; mais, dans la mesure où elles sont très importantes, l'échantillon devient alors impropre à la datation. Toutes les observations qui peuvent être faites sur le terrain à propos des possibilités de pollution doivent être soigneusement notées par les fouilleurs ; elles permettront au laboratoire soit de choisir les traitements chimiques préalables, soit d'éliminer éventuellement un échantillon pour lequel on risquerait fort d'obtenir un âge aberrant. Rappelons aussi que tout collage, tout vernissage ou tout marquage d'un os peut le polluer définitivement. L'éventuelle pollution par les matières grasses ou sucrées, par exemple, doit être indiquée.

Par ailleurs, un échantillon n'est datable que si, d'une part, son âge est dans les limites de sensibilité de la méthode, c'est-à-dire entre 200 et 35000 ans, et si, d'autre part, on n'attend pas sur l'âge une précision meilleure que ce qu'il est possible d'obtenir. En effet, il est certain qu'on a parfois historiquement connaissance de l'âge d'un objet avec une approximation meilleure que celle qui peut être fournie par une mesure de carbone 14. C'est le cas, en France, de la plupart des sites de l'époque romaine ou du Moyen Age. Pour ces périodes récentes le C-14 ne sera done utilisé que pour lever une indétermination entre deux dates assez éloignées ; citons, par exemple, le cas d'une mesure effectuée sur des charbons de bois trouvés dans les fondations d'un bâtiment du Moyen Age pour savoir si celui-ci n'a pas été construit sur les restes d'un édifice très antérieur.

Enfin l'archéologue doit s'assurer que l'échantillon collecté est en quantité suffisante : le tableau I donne les poids généralement nécessaires

\section{TABLEAU I}

Poids minimal des échantillons soumis à la datation par le $\mathrm{C}-14$.

$$
\begin{aligned}
& \text { Charbons de bois : } 10 \mathrm{~g} \text {. } \\
& \text { Tourbes : } 50 \mathrm{~g} \text {. } \\
& \text { Coquilles : } 30 \mathrm{~g} \text {. }
\end{aligned}
$$

pour qu'une mesure soit effectuée dans de bonnes conditions. Cependant le rendement en carbone

(1) On ne parlera pas ici de la datation des concrétions calcaires et des caux souterraines, qui n'est possible que si l'on connaît les proportions de carbone d'origine organique et de carbone d'orioine minérale dans les carbonates des concrétions et dans les bicarbonates dissous dans les caux. pur est assez variable, en particulier pour les os, suivant leur condition de conservation et pour la tourbe, selon qu'il s'agit de tourbe pure ou de sédiment tourbeux ; de plus il est parfois souhaitable d'effectuer des mesures de contrôle, aussi est-il préférable chaque fois que c'est possible, de doubler les poids indiqués.

\section{Précisions et interprétations des résultats.}

Les datations résultent de mesures de radioactivité ; or la radioactivité est un phénomène aléatoire et sa mesure est, de ce fait, entachée a priori d'une certaine erreur d'origine statistique (2). On essaie bien entendu, de la diminuer par l'observation du plus grand nombre possible d'événements, c'est-à-dire, ici, par le comptage du plus grand nombre possible de désintégrations $\beta$ du C-14 ; mais on est limité en pratique par la quantité de carbone qu'il est possible et commode d'utiliser dans le détecteur, et par le temps que l'on peut raisonnablement consacrer à une mesure. Aussi les âges sont-ils donnés avec des «précisions》 qui diffèrent suivant les laboratoires : elles dépendent, en effet, des caractéristiques des installations et des conditions de mesure. Dans le cas de nos installations, qui ont l'avantage de n'utiliser dans le détecteur que des quantités de carbone de l'ordre du gramme et pour une mesure d'une durée typique de 2000 minutes, on a des «précisions » qui sont de $+1300$

pour un âge de 30000 ans, par $-1500$

exemple, de \pm 500 pour 20000 ans et de \pm 90 pour 1000 ans (tableau II). En langage statistique, cela signifie que l'âge réel de l'échantillon daté a $68 \%$ de chances de se trouver dans les limites d'erreur attribuées à la mesure (par exemple, entre 910 et 1090 ans dans le cas de l'échantillon de 1000 ans) et $95,5 \%$ de chances de se trouver dans l'intervalle défini par le double de l'erreur indiqué (entre 820 et 1180 toujours dans le cas de l'échantillon de 1000 ans).

Il s'ensuit qu'il est impossible de dater, l'un par rapport à l'autre, deux événements qui sont distants dans le temps de moins de l'incertitude sur chacun des âges. Pour les mêmes raisons on peut mesurer, pour deux niveaux contemporains, des âges légèrement différents. Prenons, par exemple, le cas précis d'un gisement archéologique africain pour lequel on a obtenu les 3 résultats suivants :

GIF-1.369 prof. $1,70 \mathrm{~m} \quad: 2100 \pm 100$ ans

GIF-1.370 prof. $1,80 \mathrm{~m}: 2050 \pm 100$ ans

GIF-1.271 prof. $2,80 \mathrm{~m}: 2050 \pm 100$ ans

(2) L'erreur statistique est généralement représentée par :

$$
\sigma=\frac{\sqrt{\mathbf{N}}}{\mathrm{T}}
$$

où $\mathrm{N}$ est le nombre total de désintégrations $\mathrm{C}-14$ enregistrées pendant le temps $T$. 


\section{TABLEAU II}

Précisions relatives et précisions absolues des dates C-14 données par les appareillages les plus courants (critère 1 sigma).

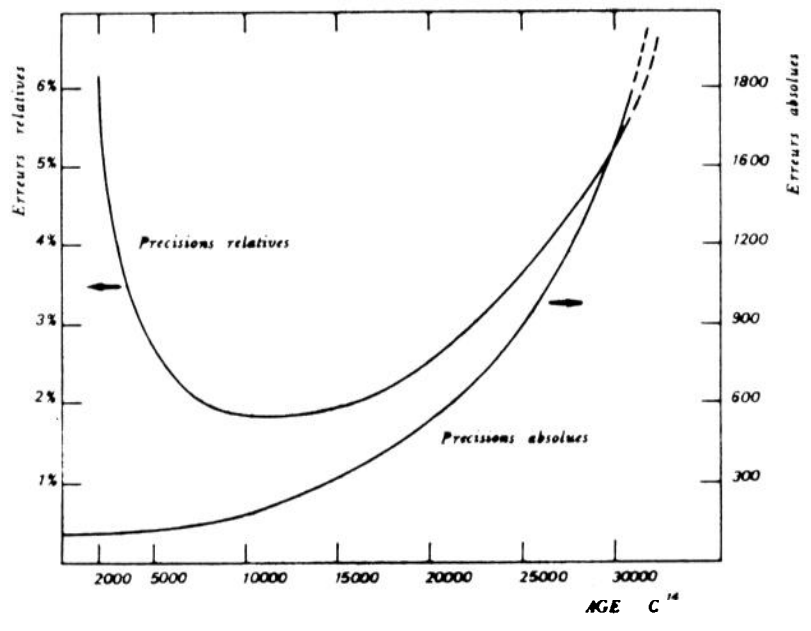

La différence d'âge entre $1,70 \mathrm{~m}$ et $1,80 \mathrm{~m}$ n'est pas significative parce qu'elle est inférieure aux erreurs statistiques qui affectent chacune des mesures. On peut plus raisonnablement conclure qu'on a ici affaire à un même âge pour les trois échantillons, bien qu'ils soient dispersés entre $1,70 \mathrm{~m}$ et $2,80 \mathrm{~m}$.

Dans des cas exceptionnels on peut cependant, en multipliant les mesures ou en augmentant leur durée, accroître la précision donnée pour un âge, mais ceci ne s'impose en fait que rarement.

Parfois une première datation conduit à un résultat tout à fait inattendu pour un échantillon que l'on croyait archéologiquement bien connu. Si on veut alors voir confirmer la mesure, il suffit de demander un contrôle au laboratoire, lui seul pourra dire si ce contrôle est justifié ou non : en effet, si l'on a trouvé, par exemple, un âge contemporain pour un os que l'on croyait néolithique, il est bien inutile de refaire une mesure sur le même os, car on a affaire à un site perturbé ; il faut revenir sur le terrain prélever d'autres échantillons. S'adresser, pour ce contrôle, à un second laboratoire non averti du problème, conduit à des mesures inutiles et peut altérer le climat de confiance qui doit régner entre les laboratoires et les chercheurs. Inutiles aussi sont les mesures demandées simultanément à plusieurs laboratoires sur un échantillon ; tous les laboratoires du monde se réfèrent à un même standard qui est un carbone moderne délivré par le National Bureau of Standard, sous forme d'acide oxalique ; chaque laboratoire s'assure périodiquement de la stabilité de rendement de ses compteurs et des caractéristiques de son électronique, et, dans les limites des erreurs statistiques, ils fourniront des résultats comparables.

On voit que le choix et le nombre des échantillons à dater, et l'interprétation des résultats posent quelquefois des problèmes qui doivent être résolus en commun par l'archéologue et le responsable du laboratoire Carbone 14.

\section{Nécessité des informations à fournir au labora- toire et publications des résultats.}

Sans insister sur l'intérêt scientifique évident pour les responsables de laboratoire de travailler sur un problème bien exposé et en collaboration étroite avec les archéologues, il est clair que des renseignements précis concernant chaque échantillon leur sont absolument indispensables : d'abord pour décider si l'on doit ou non effectuer cette étude par le C-14 et la développer, pour savoir ensuite si le site étudié n'a pas déjà fait l'objet de datations par ailleurs et pour s'assurer, enfin, que l'échantillon est bien représentatif et n'a pas subi de pollution au cours de sa conservation. Toutes ces informations sont indispensables si l'on veut obtenir une date significative et utilisable dans une étude scientifique.

Certaines demandes de renseignements surprennent quelquefois, en particulier celles qui tendent à savoir l'âge attendu par le chercheur. En réalité le fait de connaître la succession des niveaux et l'âge présumé d'un échantillon n'influence en aucune façon le résultat de la mesure. Par contre, il peut permettre de préciser ce résultat en effectuant des mesures plus prolongées et, dans le cas où il est en désaccord avec l'âge prévu de refaire ultérieurement une préparation et une mesure si toutefois l'échantillon est assez abondant.

Ce sont tous ces renseignements qui constituent la justification de la demande qui est formulée et, plus tard, la justification de la mesure effectuée. Ils sont par ailleurs indispensables lors de la publication des datations, publication qui est faite périodiquement par tous les laboratoires de datation du monde dans le journal Radiocarbon (3). L'intérêt de la publication est bien accru si l'on a pu apporter un exposé fortement documenté accompagné de références d'articles et faisant ressortir de façon claire l'importance de la datation obtenue. Celle-ci sera souvent comparée à d'autres mesures C-14. II est par suite instamment recommandé au chercheur d'envoyer un tiré à part, voire même un préprint de ses publications. On demande également de localiser avec précision le site étudié en indiquant le nom de la localité et ses coordonnées géographiques en degrés et en minutes. En général, les laboratoires envoient un questionnaire, ce qui facilite l'obtention des renseignements principaux.

De façon conventionnelle, les datations dans Radiocarbon sont accompagnées du numéro d'enregistrement de l'échantillon précédé du sigle du laboratoire (par exemple Ly-628).

(3) La revue " Radiocarbon » est publiée par l'American Journal of Science Box 2161, Yale Station, New-Haven, Connecticut. U.S.A. 
Il est bon que dans toute autre publication spécialisée faite ensuite, les âges apparaissent également accompagnés de cette référence de façon que la mesure puisse être identifiée à travers les différentes publications. Bien que ce numéro d'enregistrement permette aussi d'indiquer quel est le laboratoire qui a fait la mesure, il est préférable de mentionner en plus celui-ci, à moins que le responsable du laboratoire C-14 ne soit co-signataire de l'article.

A la suite d'un accord entre tous les laboratoires $\mathrm{C}-14$, les âges sont calculés en utilisant la période 5570 ans et en prenant comme année de référence l'année 1950 ; il en résulte que dans les âges « B.P. » (before present), le présent est en fait l'année 1950. Il est instamment recommandé de ne pas modifier ces âges lors des publications et de conserver la valeur fournie par le laboratoire ; par contre évidemment, on peut spécifier qu'elle a été calculée à partir de 1950. Un réajustement sera ainsi toujours pos- sible lorsqu'une décision générale aura été prise ì ce sujet.

On a ci-dessus rappelé la signification des erreurs qui accompagnent les mesures d'âge. C'est une information très importante et significative qui doit, dans tous les cas, être mentionnée avec l'âge lui-même, et toujours telle qu'elle a été fournie par le laboratoire de datation.

Les recommandations précédentes montrent bien que le but commun des archéologues et des responsables des laboratoires de radiocarbone est l'obtention de dates significatives et utilisables dans des études scientifiques. Ceci ne peut se faire que grâce à une collaboration sans réserves où chacun n'hésitera pas à exposer ses doutes. Des contacts fréquents et, éventuellement, des visites sur le terrain du responsable du laboratoire de datation semblent à ce sujet très souhaitables.

\section{PROGRAMME PREVU}

pour la séance du jeudi 26 octobre 1972, à 15 heures

à l'Institut de Paléontologie Humaine, 1, rue René-Panhard, Paris-13"

M. le $D^{r}$ Gruet. -- L'ossuaire semi-mégalithique de Chacé (Maine-et-Loire).

M. le $D^{r}$ Gruet et M. Glotin. - L'allée couverte de Pont-Piau-en-Champtocé (Maine-et-Loire).

M. le $D^{r}$ Gruet. - Le vase campaniforme du Gué d'Arcy (Maine-et-Loire).

M. Hinout. - Le Tardenoisien du Bassin Parisien : principe d'une méthode de classification.

M. Capitaine. - Peintures rupestres et céramiques du Hoggar.

MM. Briard, Bourhis, Onvés. - Les tumulus de l'Age du Bronze des Monts d'Arrée, Finistère, une révision.

Communication prévue pour la séance de novembre 1972 :

M. Grosjean. - Le complexe mégalithique de Settiva (Petreto-Bicchisano, Corse).

\section{SOMMAIRE}

G. Huguenin et M. Meunier. - Nouvelles stations à quartzites paléolithiques dans le département des Vosges ..................

R. DaniEL. - La grotte classique des Eyzies (dite grotte Richard),

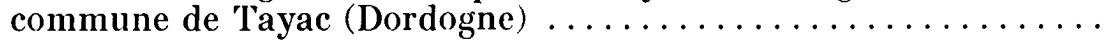

A. Debenath. - Nouvelles fouilles à Dar Es-Soltane (Champ de tir d'El Menzeh) près de Rabat (Maroc), note préliminaire ........

G. Cordier. - Instruments perforés du Loir-et-Cher ( $3^{\circ}$ supplément).

J. Arnal, J. Pfyron et A. Robert. - Les haches à ailerons en bronze de la Société Archéologique de Montpellier ...............

G. Delibrias et J. Evin. - Remarques à propos des demandes d'analyses par le Carbone 14 et l'interprétation de leurs résultats .... 